\title{
Pterostilbene induces cell cycle arrest and apoptosis in MOLT4 human leukemia cells
}

\author{
Kamila Siedlecka-Kroplewska ${ }^{1}$, Agnieszka Jozwik ${ }^{2}$, Lucyna Kaszubowska ${ }^{1}$, \\ Anna Kowalczyk ${ }^{3}$, Wojciech Boguslawski ${ }^{4}$
}

\author{
${ }^{1}$ Department of Histology, Medical University of Gdansk, Gdansk, Poland \\ ${ }^{2}$ Department of Pathophysiology, Medical University of Gdansk, Gdansk, Poland \\ ${ }^{3}$ Department of Human Histology and Embryology, University of Warmia and Masuria, Olsztyn, Poland \\ ${ }^{4}$ Department of Social and Clinical Gerontology, Medical University of Gdansk, Gdansk, Poland
}

\begin{abstract}
Pterostilbene, a polyphenolic compound present in grapes and other fruits, has been demonstrated to inhibit growth and induce apoptosis and autophagy in some cancer cell types. We found that pterostilbene at the $\mathrm{IC}_{90}$ concentration of $44 \mu \mathrm{M}$ inhibited proliferation and induced apoptosis in MOLT4 human leukemia cells. Treatment with pterostilbene resulted in a transient accumulation of cells in the $G_{0} / G_{1}$-cell cycle phase followed by the S-phase arrest. Pterostilbene-induced apoptotic death of MOLT4 cells was mediated by caspase-3 activation and was accompanied by the disruption of mitochondrial membrane potential, phosphatidylserine externalisation and internucleosomal DNA fragmentation. Our results suggest that pterostilbene could serve as a potential additional chemotherapeutic agent for the treatment of leukemia. (Folia Histochemica et Cytobiologica 2012, Vol. 50, No. 4, 574-580)
\end{abstract}

Key words: pterostilbene, $\mathrm{IC}_{90}$, polyphenols, cell cycle, caspase-3, apoptosis, mitochondrial membrane potential, ROS, MOLT4 leukemia cells

\section{Introduction}

Pterostilbene (trans-3,5-dimethoxy-4'-hydroxystilbene, Figure 1A) is a natural phytoalexin found in grapes and other fruits $[1,2]$. It has been shown to have antifungal, antioxidant, anti-inflammatory and anti-diabetic properties [3-5]. Pterostilbene is a dimethylated structural analogue (Figure 1A) of resveratrol (trans-3,4',5-trihydroxystilbene), an extensively studied constituent of wine. Despite the anti-cancer activity shown in experimental studies, the potential clinical application of resveratrol is limited due to its poor bioavailability [6]. However, pterostilbene has greater bioavailability than resveratrol [6], and the

Correspondence address: K. Siedlecka-Kroplewska, Department of Histology, Medical University of Gdansk, 1 Debinki St., 80-211 Gdansk, Poland; tel.: +48 583491437 , fax: +48 5834914 19; e-mail: ksiedlecka@gumed.edu.pl results of many studies suggest that it may be a promising chemotherapeutic agent. Pterostilbene has been found to exert antiproliferative and proapoptotic effects in various cancer types, such as lung, gastric and prostate cancer as well as in melanoma, hepatoma and leukemic cell lines [7-12]. However, the mechanisms of pterostilbene activity in cancer cell lines have not been fully elucidated. Interstingly pterostilbene has been found to induce both apoptosis and autophagy in bladder [13] and breast cancer cells [14]. Pterostilbene inhibited 12-O-tetradecanoylphorbol 13-acetate (TPA)induced invasion, migration and metastasis of human hepatoma HepG2 cells by the down-regulation of metalloproteinase-9 (MMP-9) gene expression [11]. Moreover, pterostilbene suppressed heregulin- $\beta$-mediated cell invasion, motility and cell transformation of MCF-7 human breast carcinoma cells by decreasing the MMP-9 activity [15]. It has been found that dietary administration of pterostilbene effectively reduced azoxymethane-induced formation of colonic aberrant crypt foci, pre- 
$\mathbf{A}$
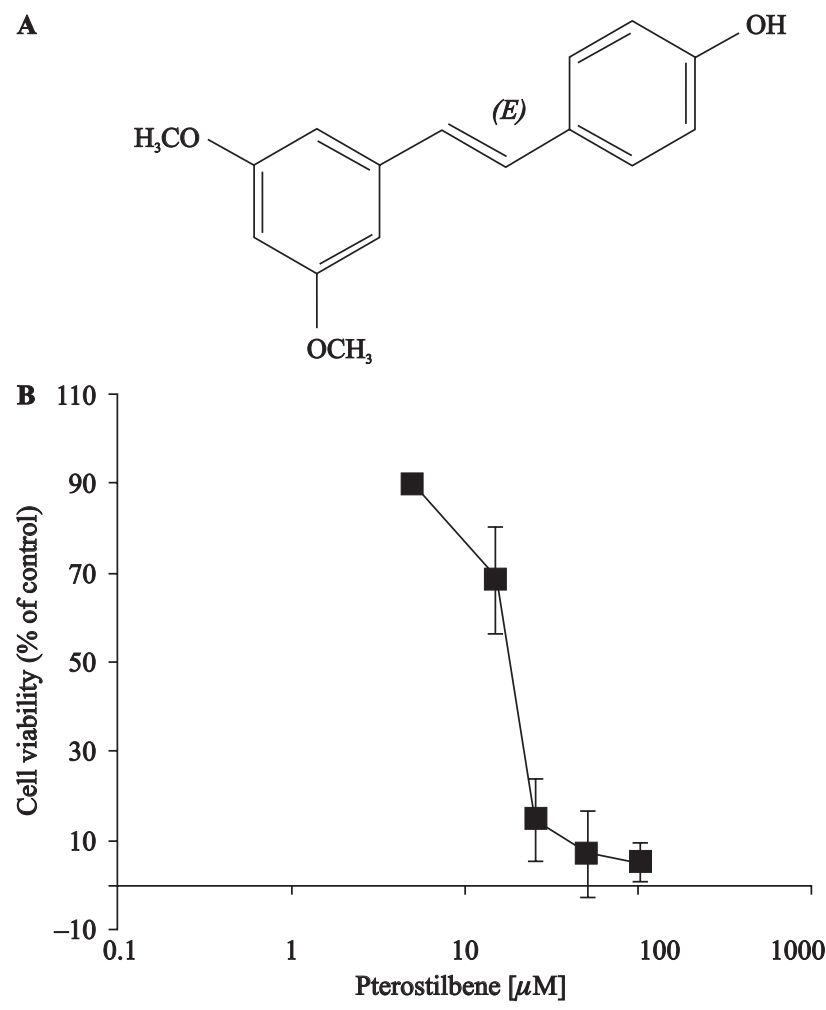

Figure 1. Chemical structure of pterostilbene (panel A). Effect of pterostilbene on viability of MOLT4 cells (panel B). MOLT4 cells were incubated for $48 \mathrm{~h}$ with increasing concentrations of pterostilbene and their viability was assessed by the neutral red uptake assay. Data is presented as means $\pm \mathrm{SD}$ of three independent experiments

neoplastic lesions and adenomas in mice [16]. Moreover, pterostilbene exerted chemopreventive effects in urethane-induced murine lung tumours [17] and inhibited 7,12-dimethylbenz[a]anthracene (DMBA)/ /TPA-induced skin tumour formation [18].

The effective action of a potential chemotherapeutic agent should result in the maximal elimination of cancer cells. However, in most studies of pterostilbene activity, the $\mathrm{IC}_{90}$ concentration classically used in toxicological studies has not been determined. Therefore, the aims of our investigation were: (i) to find the concentration of pterostilbene required to inhibit growth of MOLT4 human leukemia cells by $90 \%$ ( $\mathrm{IC}_{90}$ ), and (ii) establish mechanisms of cell death induced by pterostilbene at the $\mathrm{IC}_{90}$ concentration.

\section{Material and methods}

Chemicals. Pterostilbene was purchased from Sigma-Aldrich (USA). Pterostilbene stock solutions (200 mM) were prepared in dimethyl sulphoxide (DMSO, Sigma-Aldrich). Neutral red and RNase A were obtained from Sigma-Ald- rich (USA). Propidium iodide (PI) and H DCFDA (2', 7'-dichlorodihydrofluorescein diacetate) were purchased from Molecular Probes (USA). JC-1 (5,5',6,6'-tetrachloro1,1,3,3'-tetraethylbenzimidazolylcarbocyanine iodide) was purchased from Calbiochem (USA).

Cell culture. The MOLT4 cell line (human lymphoblastic leukemia cell line) was kindly provided by Dr. E. Augustin (Department of Pharmaceutical Technology and Biochemistry, Gdansk University of Technology, Poland). MOLT4 cells were maintained at $37^{\circ} \mathrm{C}$ in a humidified atmosphere containing 5\% $\mathrm{CO}_{2}$, in RPMI 1640 medium (Sigma-Aldrich, USA) supplemented with $10 \%$ heat-inactivated fetal bovine serum (Sigma-Aldrich, USA) and antibiotics (100 IU/mL penicillin and $100 \mu \mathrm{g} / \mathrm{mL}$ streptomycin (Sigma-Aldrich, USA).

Cytotoxicity assay. The cytotoxic effect of pterostilbene was determined by the neutral red uptake assay which is based on the ability of viable cells to incorporate the supravital dye neutral red into lysosomes. MOLT4 cells $\left(1.5 \times 10^{4} /\right.$ $/ \mathrm{mL}$ ) were exposed to pterostilbene at concentrations of between 0 and $100 \mu \mathrm{M}$ for $48 \mathrm{~h}$ (about three population doublings of MOLT4 cells). Control cells were treated with the solvent (DMSO) alone. In all experiments, the DMSO concentration was never higher than $0.1 \%(\mathrm{v} / \mathrm{v})$ and did not affect cell growth. After $48 \mathrm{~h}$ of treatment, cells were centrifuged, and washed twice with pre-warmed PBS (Phosphate-Buffered Saline, Sigma-Aldrich, USA). Supernatants were removed, cells were suspended in neutral red solution (final concentration: $33 \mu \mathrm{g} / \mathrm{mL}$ ) and then incubated for $2.5 \mathrm{~h}$ at $37^{\circ} \mathrm{C}$. Next, cells were washed twice with pre-warmed PBS, supernatants were removed and acetic acid/ethanol solution ( $1 \%$ acetic acid in $50 \%$ ethanol, $\mathrm{v} / \mathrm{v})$ was added to each sample. The absorbance was measured at $\lambda=540 \mathrm{~nm}$ using a microplate reader (Jupiter; ASYS Hitech GmbH, Austria). The number of viable cells was expressed as the percentage of control. The concentration of pterostilbene required to inhibit cell growth by $90 \%\left(\mathrm{IC}_{90}\right)$ was calculated.

Cell cycle analysis. After treatment, cells were collected, washed with cold PBS, and fixed in ice-cold 70\% ethanol overnight at $-20^{\circ} \mathrm{C}$. Thereafter, cells were washed in ice-cold PBS, suspended in staining solution $(50 \mu \mathrm{g} / \mathrm{mL}$ propidium iodide, PI, and $25 \mu \mathrm{g} / \mathrm{mL}$ DNase-free RNase A in PBS) and incubated for $30 \mathrm{~min}$ in the dark at $37^{\circ} \mathrm{C}$. Samples were analysed by flow cytometry (Becton Dickinson FACScan, USA).

Annexin V-FITC/PI assay. Phosphatidylserine externalisation was determined using an Annexin V-FITC Apoptosis Detection Kit (BD Pharmingen, USA) according to the manufacturer's protocol. After treatment, cells were washed with PBS and stained with PI and FITC-conjugated Annexin V. Samples were analysed by flow cytometry. 
Table 1. Cell cycle distribution of pterostilbene-treated MOLT4 cells

\begin{tabular}{|l|c|c|c|c|}
\hline \multirow{2}{*}{ Time of treatment $(\mathbf{h})$} & \multicolumn{3}{|c|}{ Cell cycle distribution (\% of total cell population) } \\
\cline { 2 - 5 } & $\mathbf{S u b}_{\mathbf{1}} \pm \mathbf{S D}$ & $\mathbf{G}_{\mathbf{0}} / \mathbf{G}_{\mathbf{1}} \pm \mathbf{S D}$ & $\mathbf{S} \pm \mathbf{S D}$ & $\mathbf{G}_{\mathbf{2}} / \mathbf{M} \pm \mathbf{S D}$ \\
\hline Control & $0.5 \pm 0.1$ & $37.6 \pm 1.2$ & $40.3 \pm 0.8$ & $22.1 \pm 1.0$ \\
\hline $6 \mathrm{~h}$ & $1.2 \pm 1.0$ & $47.4 \pm 5.6^{*}$ & $31.3 \pm 3.4$ & $13.8 \pm 0.2$ \\
\hline $12 \mathrm{~h}$ & $1.5 \pm 1.0$ & $44.4 \pm 1.8$ & $41.8 \pm 1.4$ & $12.9 \pm 0.5$ \\
\hline $24 \mathrm{~h}$ & $10.5 \pm 5.5$ & $37.2 \pm 0.5$ & $44.7 \pm 5.2^{*}$ & $7.7 \pm 0.8$ \\
\hline $48 \mathrm{~h}$ & $29.4 \pm 2.2^{*}$ & $23.7 \pm 5.9$ & $26.6 \pm 1.5$ & $21.0 \pm 2.5$ \\
\hline
\end{tabular}

Data is presented as means \pm SD of three independent experiments; *Statistically significant difference vs. respective fraction in control cells, $\mathrm{p}<0.05$

DNA fragmentation analysis. DNA fragmentation was examined as described previously [19]. DNA fragments were fractionated by electrophoresis on $1.8 \%$ agarose gels, stained with ethidium bromide, and photographed using Gel Doc 2000 (Bio-Rad, Italy).

Caspase-3 activity assay. Caspase-3 activity was measured using a FITC-conjugated Monoclonal Active Caspase3 Antibody Apoptosis Kit I (BD Pharmingen, USA) according to the manufacturer's protocol. After treatment, cells were stained with FITC-conjugated anti-active caspase-3 antibody. Samples were analysed by flow cytometry.

Measurement of mitochondrial membrane potential. Analysis of mitochondrial membrane potential was performed using the JC-1 dye as described previously [19]. Samples were analysed by flow cytometry.

Detection of reactive oxygen species. The intracellular production of reactive oxygen species (ROS) was analysed by flow cytometry. Cells were exposed to pterostilbene or DMSO (control). 30 min before the end of incubation, $\mathrm{H}_{2}$ DCFDA was added at a final concentration of $10 \mu \mathrm{M}$. Cells were then washed, suspended in cold PBS, and analysed for DCF fluorescence by flow cytometry.

Statistical analysis. Statistical analysis was performed using Statistica 9 software (Statsoft, Poland). Data is expressed as means \pm SD. Statistical differences were evaluated using the Mann-Whitney U test. Differences were considered significant at $\mathrm{p}<0.05$.

\section{Results}

\section{Effect of pterostilbene on cell viability and cell cycle}

The treatment of MOLT4 cells with pterostilbene for $48 \mathrm{~h}$ resulted in a dramatic decrease in cell viability as assessed by neutral red uptake assay (Figure 1B). The estimated pterostilbene concentration required to inhibit growth of MOLT4 cells by $90 \%\left(\mathrm{IC}_{90}\right)$ was $44 \mu \mathrm{M}$.

The cell cycle analysis revealed that after $6 \mathrm{~h}$ of incubation of MOLT4 cells with pterostilbene at the $\mathrm{IC}_{90}$ concentration, there was a statistically significant increase in the number of cells in the $G_{0} / G_{1}$ phase (Table 1, Figure 2). However, this effect was transient. After $24 \mathrm{~h}$ of incubation with the compound, there was a significant accumulation of cells in the S-phase, which was accompanied by a corresponding decrease in the number of cells in $\mathrm{G}_{0} / \mathrm{G}_{1}$ and $\mathrm{G}_{2} / \mathrm{M}$ fractions (Table 1 ). Treatment of cells with $44 \mu \mathrm{M}$ pterostilbene for $48 \mathrm{~h}$ led to a significant increase of the population of cells in the sub-G1 fraction (Table 1, Figure 2).

\section{Pterostilbene-induced apoptosis of MOLT4 cells}

Phosphatidylserine externalisation, indicative of the loss of plasma membrane asymmetry, is considered to be a hallmark of apoptosis. Treatment of MOLT4 cells with $44 \mu \mathrm{M}$ pterostilbene resulted in a time-dependent statistically significant increase in the number of early apoptotic and late apoptotic/necrotic cells (Figures 3A, B). After $12 \mathrm{~h}$ of pterostilbene treatment, Annexin- $\mathrm{V}^{+} / \mathrm{PI}^{-}$cells (early apoptotic) and Annexin$\mathrm{V}^{+} / \mathrm{PI}^{+}$cells (late apoptotic/necrotic) constituted about $5 \%$ and $9 \%$ of the total measured cell population, respectively. After $24 \mathrm{~h}$ of incubation with pterostilbene, the fractions of Annexin- $\mathrm{V}^{+} / \mathrm{PI}^{-}$and Annexin- $\mathrm{V}^{+} / \mathrm{PI}^{+}$cells were $16 \%$ and $14 \%$, respectively. The number of early apoptotic and late apoptotic/necrotic cells further increased after $48 \mathrm{~h}$ of pterostilbene treatment: early apoptotic cells constituted about $20 \%$, whereas late apoptotic/necrotic made up $22 \%$ of the total measured cell population (Figures 3A, B).

Since internucleosomal DNA fragmentation is a typical marker of the late phase of apoptosis, we examined the effect of pterostilbene on DNA degradation in MOLT4 cells. As shown in Figure 3C, after $12 \mathrm{~h}$ and $24 \mathrm{~h}$ of treatment with $44 \mu \mathrm{M}$ pterostilbene, 


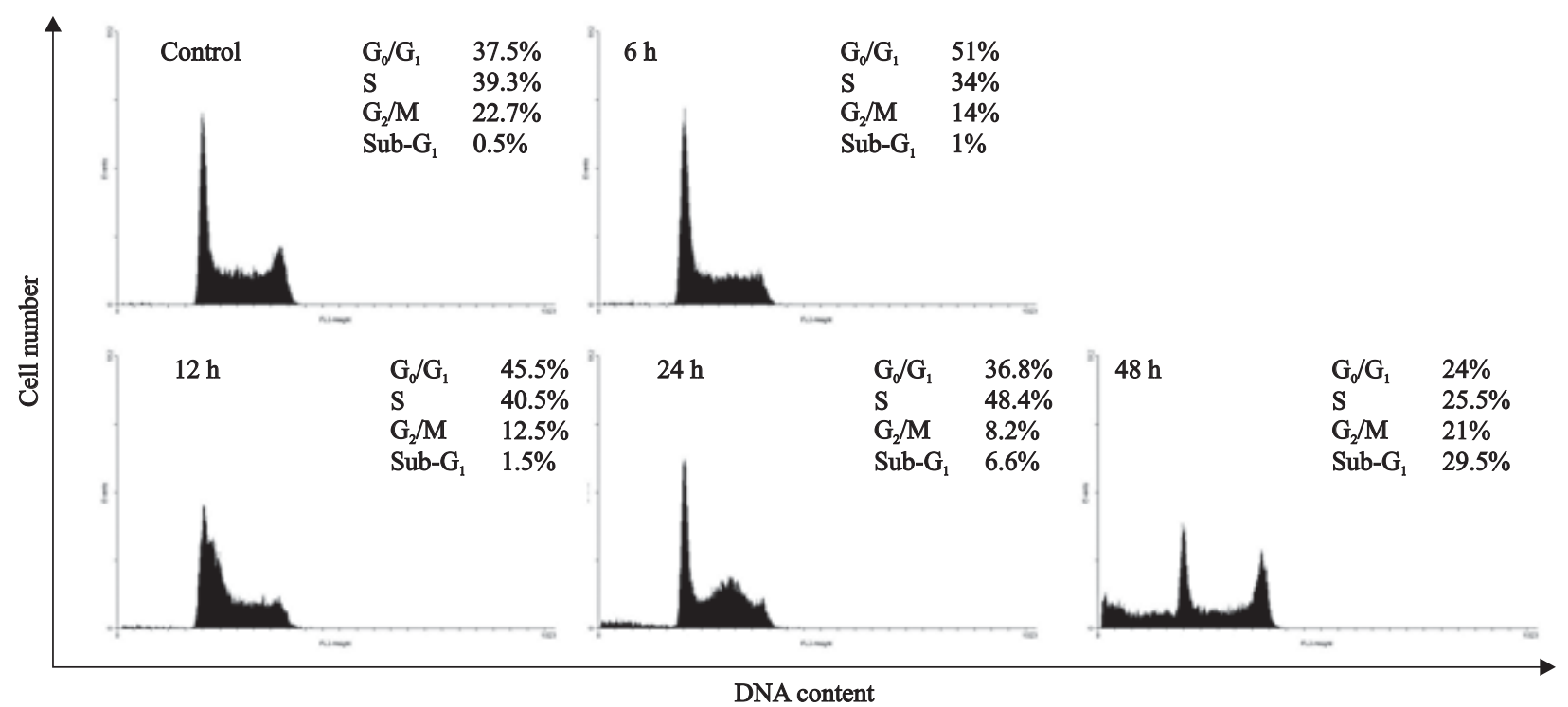

Figure 2. Cell cycle analysis. MOLT4 cells were treated with $44 \mu \mathrm{M}$ pterostilbene for $6,12,24$, and $48 \mathrm{~h}$; control untreated cells (flow cytometry analysis, PI staining). Data is representative of three independent experiments which gave similar results

only slight changes were observed compared to control, i.e. untreated, cells. An apoptotic ladder-like pattern was clearly visible only after $48 \mathrm{~h}$ of incubation with the compound.

The activity of caspase- 3 is required for the executive phase of apoptosis. As shown in Figure 3D, $44 \mu \mathrm{M}$ pterostilbene led to a statistically significant increase in the percentage of cells with active caspase-3. After $48 \mathrm{~h}$ of treatment, caspase- 3 activation was detected in $32 \%$ of the total measured cell population.

Mitochondria play an important role in the intrinsic apoptotic pathway. The decrease/loss of mitochondrial membrane potential related to mitochondrial dysfunction is considered to be an apoptotic event. Therefore, we examined the effect of pterostilbene on changes of mitochondrial membrane potential in MOLT4 cells. Results, shown in Figure 3D, demonstrate that $44 \mu \mathrm{M}$ pterostilbene induced statistically significant changes of the mitochondrial membrane potential. After $48 \mathrm{~h}$ of incubation with the compound, about $20 \%$ of cells showed reduced mitochondrial potential.

\section{Effect of pterostilbene on intracellular ROS production}

To elucidate whether pterostilbene-induced death of MOLT4 cells is accompanied by oxidative stress, we examined the effect of this polyphenolic compound on intracellular ROS (reactive oxygen species) production. As shown in Figure 4, compared to untreat- ed control cells, $4 \mathrm{~h}$ and $6 \mathrm{~h}$ of treatment with $44 \mu \mathrm{M}$ pterostilbene did not change the formation of ROS in MOLT4 cells. DCF fluorescence, representing the overall intracellular ROS production, was not altered in pterostilbene-treated cells compared to control cells.

\section{Discussion}

Recently, many in vitro and in vivo studies have indicated that pterostilbene may be a promising chemotherapeutic agent [7-18]. In the present study, we have presented novel observations that pterostilbene, a natural resveratrol analogue, induced cell cycle arrest and apoptosis in MOLT4 human leukemia cells.

Our results revealed that treatment of MOLT4 cells for $6 \mathrm{~h}$ with pterostilbene at a biologically significant $\mathrm{IC}_{90}$ concentration resulted in a transient accumulation of cells in the $G_{0} / G_{1}$ phase, followed by S-phase cell cycle arrest after prolonged incubation with the compound. Pterostilbene-induced $\mathrm{G}_{0} / \mathrm{G}_{1}$ cell cycle arrest has also been observed in AGS human gastric cancer cells [8] as well as in LNCaP human androgen-responsive prostate cancer cells [9]. Pterostilbene has been found to cause S-phase cell cycle arrest in MCF-7 human breast cancer cells [20] and in HL60 human leukemia cells [12].

Apoptosis is a genetically programmed cell death that plays a crucial role in both the development and maintenance of tissue homeostasis. Chemical compounds that affect apoptotic pathways and eliminate 
A

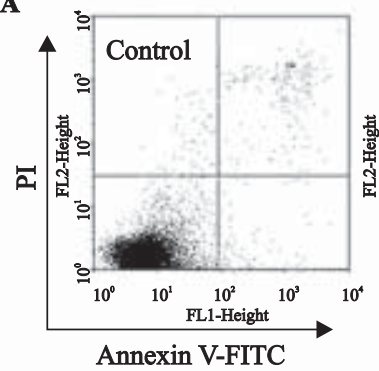

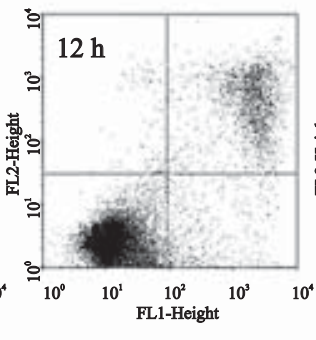
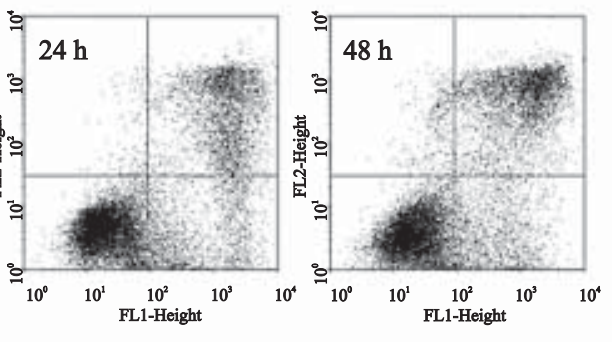

C
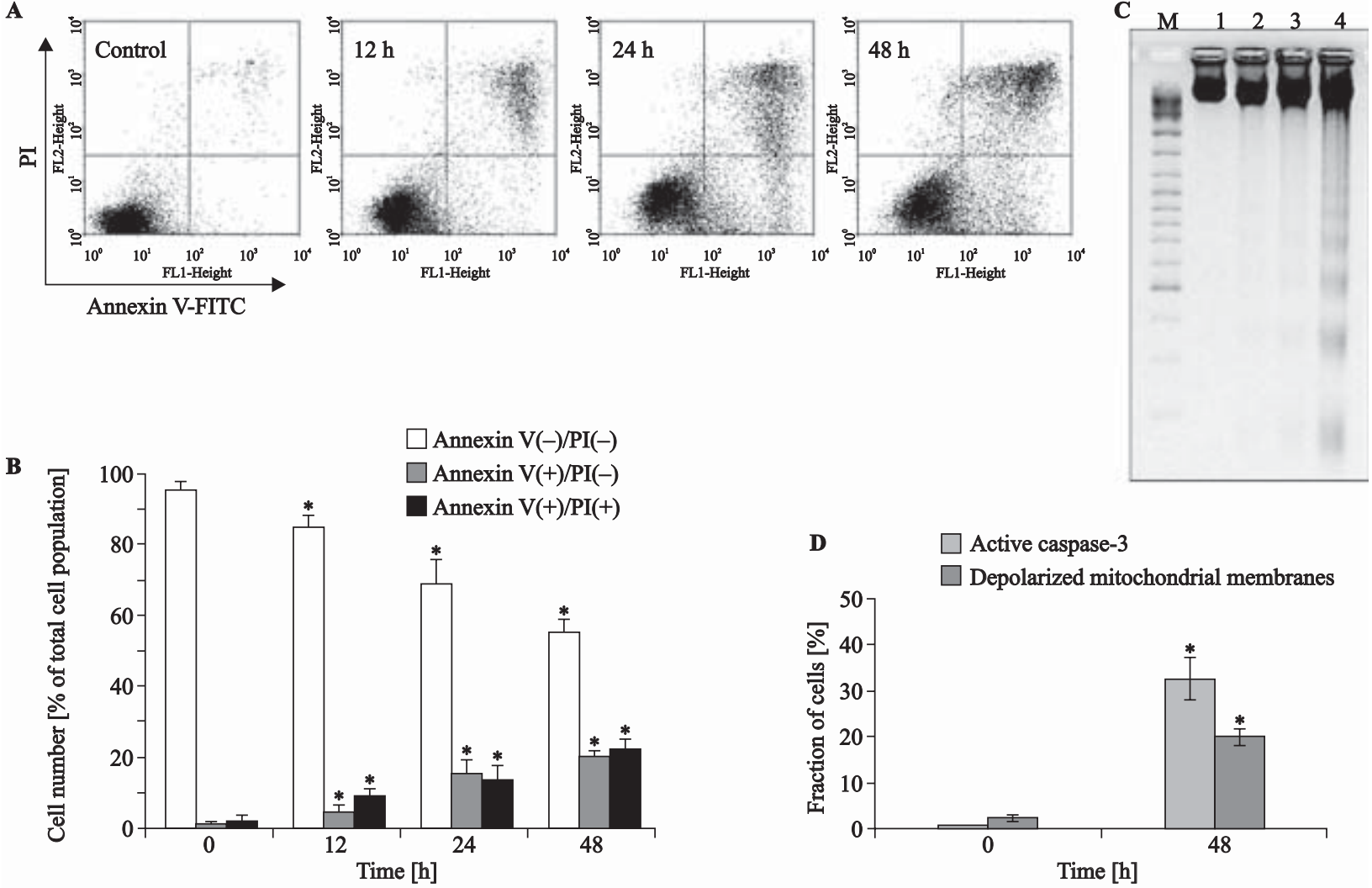

D

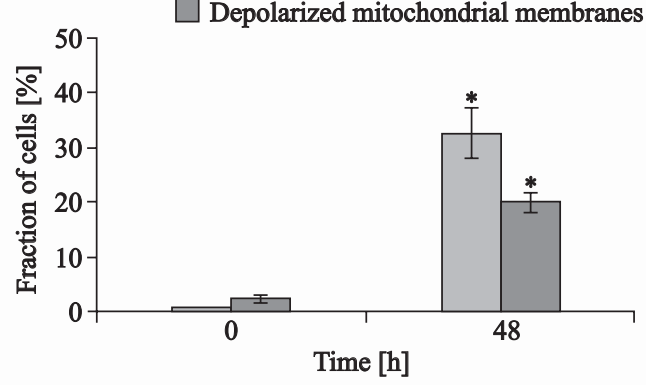

Figure 3. Detection of apoptosis in pterostilbene-treated MOLT4 cells. (A) Time-course changes of phosphatidylserine externalisation induced by pterostilbene in MOLT4 cells (flow cytometry analysis, Annexin V-FITC/PI staining). MOLT4 cells were incubated with $44 \mu \mathrm{M}$ pterostilbene for 0-48 h. Data is representative of three independent experiments. The bottom right quadrant of each dot plot represents Annexin V positive/PI negative cells (Annexin $\mathrm{V}^{+} / \mathrm{PI}^{-}$) corresponding to early apoptotic cells, whereas the upper right quadrant represents Annexin V positive/PI positive (Annexin $\mathrm{V}^{+} / \mathrm{PI}^{+}$) cells corresponding to late apoptotic/necrotic cells. (B) Percentage of apoptotic cells (exhibiting phosphatidylserine externalisation) induced by pterostilbene (flow cytometry analysis, Annexin V-FITC/PI staining). MOLT4 cells were incubated with $44 \mu \mathrm{M}$ pterostilbene for $0-48 \mathrm{~h}$. Data is presented as means \pm SD of three independent experiments.

* statistically significant difference compared to the respective data of control (untreated cells), p < 0.05. (C) DNA fragmentation induced by pterostilbene. MOLT4 cells were incubated with $44 \mu \mathrm{M}$ pterostilbene for $0-48 \mathrm{~h}$. M, 100-10,000 bp DNA marker; lane 1, control (untreated cells); lanes 2-4, 12, 24, and $48 \mathrm{~h}$ of pterostilbene treatment, respectively. DNA degradation was analysed by agarose gel electrophoresis. Data is representative of three independent experiments. (D) Effect of pterostilbene on caspase-3 activation and changes of mitochondrial membrane potential. MOLT4 cells were treated with $44 \mu \mathrm{M}$ pterostilbene for $48 \mathrm{~h}$. Caspase- 3 activation was determined by flow cytometry after staining with antiactive caspase- 3 antibody. Changes in mitochondrial membrane potential were analysed by flow cytometry after JC-1 staining. Data is presented as means \pm SD of three independent experiments. * statistically significant difference compared to the respective data of control (untreated) cells, $\mathrm{p}<0.05$

cancer cells are considered to be promising anticancer drugs. In pterostilbene-treated MOLT4 cells, several markers of apoptosis were detected. Pterostilbene induced time-dependent changes in phosphatidylserine externalisation which belongs to early apoptotic events. Similar results have been shown in pterostilbene-treated K562 human leukemia cells [12]. Additionally, the induction of apoptotic cell death in MOLT4 cells treated by pterostilbene at the $\mathrm{IC}_{90}$ concentration was confirmed by the detection of inter- nucleosomal DNA fragmentation. These findings were supported by the results of the cell cycle analysis, since the proportion of cells in the sub-G1 fraction, indicative of apoptotic DNA cleavage, increased significantly after $48 \mathrm{~h}$ of pterostilbene treatment. Pterostilbene-induced apoptotic DNA fragmentation has also been detected in MCF-7 and MDA-MB-231 human breast cancer cells [20], NCI-H460 and SKMES-1 human lung cancer cells [7] as well as in AGS human gastric cancer cells [8]. 


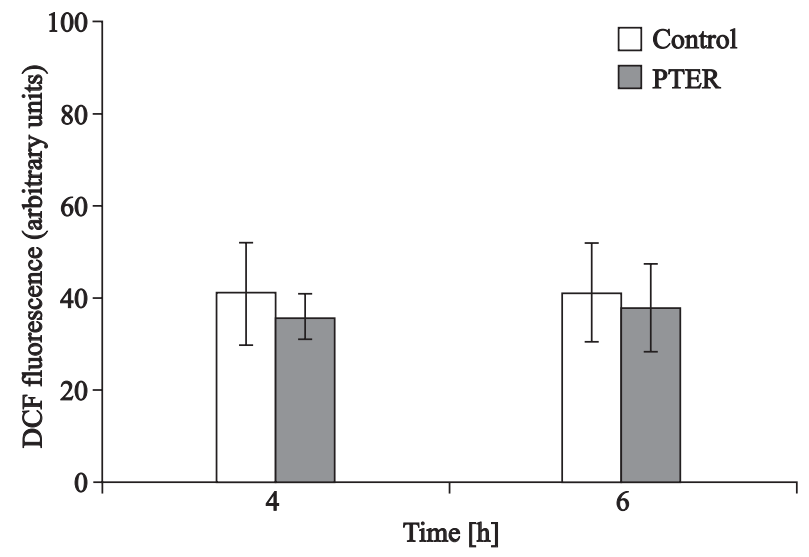

Figure 4. Effect of pterostilbene on the intracellular production of reactive oxygen species. MOLT4 cells were exposed to $44 \mu \mathrm{M}$ pterostilbene (PTER) for $4 \mathrm{~h}$ and $6 \mathrm{~h}$. Cells were analysed for DCF fluorescence by flow cytometry. Data is presented as means \pm SD of three independent experiments. There were no statistically significant differences between control and pterostilbene-treated cells

Similarly to our observations, caspase- 3 dependent apoptosis has been found in other pterostilbene-treated cancer cell lines such as SK-MEL-2 and MeWo human melanoma cells [10], NCI-H460 and SK-MES-1 human lung cancer cells [7] as well as AGS human gastric cancer cells [8]. The finding in the present study of the disruption of mitochondrial membrane potential by pterostilbene at the $\mathrm{IC}_{90}$ concentration was indicative of the intrinsic apoptotic pathway activation in MOLT4 cells. Mitochondrial depolarisation was also detected in pterostilbene-treated MCF-7 and MDA-MB-231 human breast cancer cells [20].

Despite exhibiting antioxidant properties [4], pterostilbene has been shown to induce oxidative stress by increasing superoxide anion levels in MCF7 and MDA-MB-231 breast cancer cell lines [20]. Our results suggest that changes of ROS levels in pterostilbene-treated cancer cells may depend on the type of tested cells, since we did not observe oxidative stress in MOLT4 cells.

In conclusion, we demonstrated that pterostilbene at the $\mathrm{IC}_{90}$ concentration was able to inhibit proliferation and induce apoptosis of MOLT4 human lymphoblastic leukemia cells. The results of this study suggest that this polyphenolic compound could serve as a potential additional chemotherapeutic agent for the treatment of leukemia.

\section{Acknowledgements}

This work was supported by grant No. W-110/20062008 from the Medical University of Gdansk, Poland, and by grant No. N N204 132040 from the Polish Ministry of Science and Higher Education. We thank Professor J.M. Witkowski and Professor E. Bryl (Department of Pathophysiology, Medical University of Gdansk, Poland) for the availability of the flow cytometry laboratory and their expert advice.

\section{References}

1. Adrian M, Jeandet P, Douillet-Breuil AC, Tesson L, Bessis $\mathrm{R}$. Stilbene content of mature Vitis vinifera berries in response to UV-C elicitation. J Agric Food Chem. 2000;48:6103-6105.

2. Rimando AM, Kalt W, Magee JB, Dewey J, Ballington JR. Resveratrol, pterostilbene, and piceatannol in vaccinium berries. J Agric Food Chem. 2004;52:4713-4719.

3. Pezet R, Pont V. Ultrastructural observations of pterostilbene fungitoxicity in dormant conidia of Botrytis cinerea Pers. J Phytopathol. 1990;129:19-30.

4. Rimando AM, Cuendet M, Desmarchelier C, Mehta RG, Pezzuto JM, Duke SO. Cancer chemopreventive and antioxidant activities of pterostilbene, a naturally occurring analogue of resveratrol. J Agric Food Chem. 2002;50:3453-3457.

5. Pari L, Satheesh MA. Effect of pterostilbene on hepatic key enzymes of glucose metabolism in streptozotocin- and nicotinamide-induced diabetic rats. Life Sci. 2006;79:641-645.

6. Kapetanovic IM, Muzzio M, Huang Z, Thompson TN, McCormick DL. Pharmacokinetics, oral bioavailability, and metabolic profile of resveratrol and its dimethylether analog, pterostilbene, in rats. Cancer Chemother Pharmacol. 2011;68:593-601.

7. Schneider JG, Alosi JA, McDonald DE, McFadden DW. Pterostilbene inhibits lung cancer through induction of apoptosis. J Surg Res. 2010;161:18-22.

8. Pan MH, Chang YH, Badmaev V, Nagabhushanam K, Ho CT. Pterostilbene induces apoptosis and cell cycle arrest in human gastric carcinoma cells. J Agric Food Chem. 2007;55:7777-7785.

9. Wang TT, Schoene NW, Kim YS, Mizuno CS, Rimando AM. Differential effects of resveratrol and its naturally occurring methylether analogs on cell cycle and apoptosis in human androgen-responsive LNCaP cancer cells. Mol Nutr Food Res. 2010;54:335-344.

10. Schneider JG, Alosi JA, McDonald DE, McFadden DW. Effects of pterostilbene on melanoma alone and in synergy with inositol hexaphosphate. Am J Surg. 2009;198:679-684.

11. Pan MH, Chiou YS, Chen WJ, Wang JM, Badmaev V, Ho CT. Pterostilbene inhibited tumor invasion via suppressing multiple signal transduction pathways in human hepatocellular carcinoma cells. Carcinogenesis. 2009;30:1234-1242.

12. Tolomeo M, Grimaudo S, Di Cristina A. et al. Pterostilbene and 3'-hydroxypterostilbene are effective apoptosis-inducing agents in MDR and BCR-ABL-expressing leukemia cells. Int J Biochem Cell Biol. 2005;37:1709-1726.

13. Chen RJ, Ho CT, Wang YJ. Pterostilbene induces autophagy and apoptosis in sensitive and chemoresistant human bladder cancer cells. Mol Nutr Food Res. 2010;54:1819-1832.

14. Wang Y, Ding L, Wang X et al. Pterostilbene simultaneously induces apoptosis, cell cycle arrest and cyto-protective autophagy in breast cancer cells. Am J Transl Res. 2012;4:44-51.

15. Pan MH, Lin YT, Lin CL, Wei CS, Ho CT, Chen WJ. Suppression of Heregulin- $31 /$ HER2-Modulated Invasive and Aggressive Phenotype of Breast Carcinoma by Pterostilbene via Inhibition of Matrix Metalloproteinase-9, p38 Kinase Cascade and Akt Activation. Evid Based Complement Alternat Med. 2011;2011:562187. 
16. Chiou YS, Tsai ML, Wang YJ et al. Pterostilbene inhibits colorectal aberrant crypt foci (ACF) and colon carcinogenesis via suppression of multiple signal transduction pathways in azoxymethane-treated mice. J Agric Food Chem. 2010;58: 8833-8841.

17. Chen RJ, Tsai SJ, Ho CT et al. Chemopreventive Effects of Pterostilbene on Urethane-Induced Lung Carcinogenesis in Mice via the Inhibition of EGFR-Mediated Pathways and the Induction of Apoptosis and Autophagy. J Agric Food Chem. 2012 (in press).

18. Tsai ML, Lai CS, Chang YH, Chen WJ, Ho CT, Pan MH. Pterostilbene, a natural analogue of resveratrol, potently in- hibits 7,12-dimethylbenz[a]anthracene (DMBA)/12-O-tetradecanoylphorbol-13-acetate (TPA)-induced mouse skin carcinogenesis. Food Funct. 2012;3:1185-1194.

19. Augustin E, Moś-Rompa A, Skwarska A, Witkowski JM, Konopa J. Induction of G2/M phase arrest and apoptosis of human leukemia cells by potent antitumor triazoloacridinone C-1305. Biochem Pharmacol. 2006;72:1668-1679.

20. Alosi JA, McDonald DE, Schneider JS, Privette AR, McFadden DW. Pterostilbene inhibits breast cancer in vitro through mitochondrial depolarization and induction of caspase-dependent apoptosis. J Surg Res. 2010;161: 195-201.

Submitted: 24 November, 2012 Accepted after reviews: 29 November, 2012 